\title{
I Feel Something in My Eye; A Retrospective Study on the Outcome of Patients with Corneal Foreign Bodies Presenting to Emergency Department
}

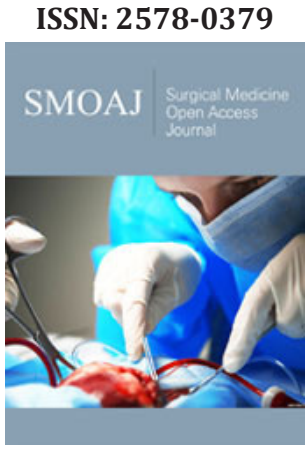

*Corresponding author: Dr. Pourya Pouryahya MD, FACEM, GC ClinEpi, CCPU, M Trauma, MPH Emergency Medicine Consultant, Department of Emergency Medicine, Casey Hospital, Monash Health Adjunct Lecturer, School of Clinical Sciences, Department of Medicine, Monash university Director of Emergency Medicine Research (DEMR), Casey hospital, Monash Health 62-70 Kangan drive, Berwick, Victoria 3806, Australia

T: +61 (03) 87681869

E: Pourya.Pouryahya@monashhealth.org

Submission: 監 October 19, 2020

Published: 眥 November 30, 2020

Volume 3 - Issue 5

How to cite this article: POURYAHYA P, Meyer A, Ling Z, Ooi A. I Feel Something in My Eye; A Retrospective Study on the Outcome of Patients with Corneal Foreign Bodies Presenting to Emergency Department. Surg Med Open Acc J. 3(5). SMOAJ.000573. 2020. DOI: 10.31031/SMOAJ.2020.03.000573

Copyright@ POURYAHYA P, This article is distributed under the terms of the Creative Commons Attribution 4.0 International License, which permits unrestricted use and redistribution provided that the original author and source are credited.

\author{
POURYAHYA $\mathrm{P}^{1,2,3 *}$, Meyer $\mathrm{A}^{1,2,3}$, Ling $\mathrm{Z}^{3}$ and Ooi $\mathrm{A}^{3}$ \\ ${ }^{1}$ Casey hospital, Emergency Department, Program of Emergency Medicine, Monash Health, \\ Melbourne, Australia \\ ${ }^{2}$ Monash Emergency Research Collaborative (MERC), Program of Emergency Medicine, \\ Monash Health, Melbourne, Australia
}

${ }^{3}$ Faculty of Medicine, Nursing and Health Sciences, Monash University, Melbourne, Australia

\begin{abstract}
Introduction:Extraocular foreign bodies (EOFBs) are a common presentation to the emergency department (ED). Given that inadequate management can result in severe complications including visual impairment, ED clinicians may be overly cautious and often schedule patient reviews in the ED even where it is unnecessary, placing a burden on hospital resources.

Objective: This study aims to identify potential risk factors in predicting re-presentation of patients with EOFBs following ED management. This will not only help in providing better patient outcomes but also reduce the burden on hospital resources.
\end{abstract}

Method: A retrospective data analysis of patients who presented to three Monash Health EDs between June and December 2016 with EOFBs was performed. Characteristics of cases where patients presented with corneal EOFBs including the size and material of EOFB, visual acuity at initial presentation and outcome of follow-ups were reviewed and analysed.

Result: 123 patients presented with corneal foreign bodies, of which 3 were referred directly to the ophthalmology clinic after initial ED assessment for removal and post-removal management by ophthalmologist. 77 patients were managed in the ED and discharged with no planned reviews, of which 2 represented with complications. 31 patients were scheduled for review in ED 24 to 72 hours post-discharge, and 10 patients were referred directly to the ophthalmology clinic for follow up post ED discharge.

Conclusion: The study demonstrated that the rate of re-presentation for patients with corneal foreign bodies is higher if the EOFB is metallic, the size is smaller or visual acuity is abnormal on initial presentation.

Keywords: Foreign body;Ocular;External ocular foreign body;Corneal foreign body; Emergency department; Ophthalmology

\section{Introduction}

Ocular trauma is a common presentation to emergency departments (ED) [1] and ocular foreign bodies are significant contributors to these ophthalmic emergencies, accounting for $5 \%$ of all ophthalmology consultations in the emergency department [2]. Ocular foreign bodies can result in open globe lacerations in the form of intraocular foreign bodies (IOFB) or remain superficial, causing closed globe injuries as extraocular foreign bodies (EOFB) [3]. While closed globe traumas including those caused by EOFBs are less severe than open globe traumas and usually result in better visual outcomes [4], they still represent a significant disease burden in the ED. In the assessment of ocular injuries, visual acuity (VA), despite being subjective due to its physiologic nature remains a reliable indicator of the severity of injury and predictor of visual outcome [5]. The severity of an EOFB is dependent on the location where it is lodged, with the zones of injury determined via the globe opening's most posterior full-thickness aspect [3]. A conjunctival foreign body is unlikely to cause a decrease in visual acuity [6], given that the conjunctiva is a protective moisturizing lining covering the sclera. A 
sub-tarsal foreign body has the potential to cause corneal damage and disrupt vision, but given that it, like conjunctival foreign bodies exists outside of the zones of injury, it can be easily localized after eyelid eversion and removed with mechanical methods such as using a cotton [7].

On the other hand, corneal foreign bodies which can be classified as 'zone I' injuries, usually warrant a more prudent approach involving Emergency physicians or ophthalmologist. Patients may present with a sudden onset of persistent eye discomfort, accompanied by ocular inflammatory responses consisting of erythema, lacrimation, blepharospasm, photophobia, blurred vision and potential visual acuity deficit following a highrisk activity. Failed or incomplete removal of corneal foreign bodies can result in infections, visual disturbance, corneal epithelial injury and corneal abrasions, a leading cause of ocular trauma in the ED. In addition, corneal foreign bodies are of a significant burden in the ED, with about $30 \%$ of patients presenting with eye injuries [8-10]. To the best of our knowledge, the risk profile of post-treatment complications associated with EOFBs, particularly with corneal foreign bodies, has not been well-established. Consequently, patients are over-cautiously asked to represent to ED for review or referred to ophthalmologist. This adds unnecessary burden on hospital resources which could otherwise be minimized by referral to appropriate departments for follow-ups according to an established risk assessment. This retrospective study aimed to identify the risk factors in patients with corneal EOFB developing complications or having unresolved conditions following diagnosis and management in the ED.

\section{Methods}

This was a retrospective cross-sectional study of patients presenting to any of three Monash Health EDs between June 2016 and December 2016 with a suspected extraocular foreign body (EOFB). Monash Health, located in south-east Melbourne, is the largest health network in Victoria, Australia, with approximately 230,000 annual presentations across three hospitals: Monash Medical Centre (tertiary hospital), Dandenong Hospital and Casey Hospital (district hospital). This study was approved by the Monash Health and Monash University Human Research and Ethics Committees (RES-19-0000-495Q). Cases were identified through the ED medical records (Symphony, EMIS Health, Leads, UK) between June 2016 and December 2016 by electronically filtering for patients presented with ophthalmological emergencies including eye injuries, visual disturbance, ocular FB, etc. or referred to Ophthalmology team during their ED stay. Data obtained included patient demographics of age and sex, eye(s) involved and visual acuity at the time of presentation. The size, nature, and location of EOFBs were also identified where the data was documented. The outcome of managed EOFB in both initial presentation and subsequent re-presentations reviewed to uncover possible risk factors for complications or re-presentations. From a total of 224 EOFB identified cases, 101 were excluded as they were diagnosed with superficial and non-corneal EOFBs such as in the sub-tarsal plate or conjunctiva. These patients were managed in the ED without subsequent complications.

For the purposes of the study, re-presentation was defined as a patient whose condition was unresolved or who developed complications following initial management and discharge from the ED. The percentage of patients who re-presented was derived for the following possible risk factors: Patient age, visual acuity (VA) at initial presentation, EOFB material and size. Patients were excluded from the study if the contents of their records did not have specific details and could not be reliably categorized. VA at initial presentation was considered for the affected eye(s). For the size of EOFB, where descriptive terms were used in the patient records, size estimations were applied as follows: "large", "small" and "macroscopic" were regarded as size >1-2 mm, "dot", "spec", "tiny" and "microscopic" were regarded as size $\leq 1-2 \mathrm{~mm}$.

The results were then compared to determine if those risk factors resulted in a significantly higher chance of re-presentation. Chi square analysis was used to examine if there is a significant difference for risk in re-presentation within the different categories of the possible risk factors, with the concept that a lack of a significant difference would result in an even distribution of case re-presentation across the possible risk factors. The documented VA of patients with abnormal VA in the eye affected by EOFB at initial presentation was converted to LogMAR (Log of Minimum Angle of Resolution) values [11]. The mean VA of patients in this group who re-presented and did not re-present was compared with a t-test to determine whether the risk of re-presentation is increased by a particular range of VA results at initial presentation.

\section{Result}

Total ED presentations between June and December 2016 were 129205 cases, with $0.173 \%$ of total cases having a chief complaint of foreign body sensation in the eye. A total of 123 patients with corneal EOFBs were included in the study, with a mean age of 40.4 \pm 13.8 years (range 16.7-70.3 years, interquartile range 28.849.6 years). 119 of the patients (96.7\%) were male and 4 (3.3\%) were female (Table 1). 120 out of the 123 patients were managed in the ED, while the other 3 required ophthalmology consults for embedded corneal FBs within the stroma. 1 required FB removal in the operating theatre and the other 2 under local anesthesia by the ophthalmologist. 77 patients out of 120 who were managed in ED, were advised to see their GP for review within 24-48 hours, or return to ED if they had any concerns, of which 2 re-presented with ongoing symptoms and complications; 1 with infective keratitis with worsening VA, and the other for a small residual FB, complicated by conjunctivitis. 31 patients were scheduled for re-assessment and review in ED within 48-72 hours, of which 11 did not attend. 10 of these patients required further management including residual EOFB/rust ring removal, and 4 were referred to Ophthalmology clinic due to ongoing symptoms, complications, or failed attempt of residual EOFB/rust ring removal. The remaining 12 patients required urgent or semi urgent ophthalmology consult on arrival 
for failed attempts in EOFB removal or complications including superimposed infection; mainly conjunctivitis or bacterial keratitis, which was subsequently managed by ophthalmologists (Figure 1).

\section{Subgroup Analysis of Re-presentation Group (Figure 1)}

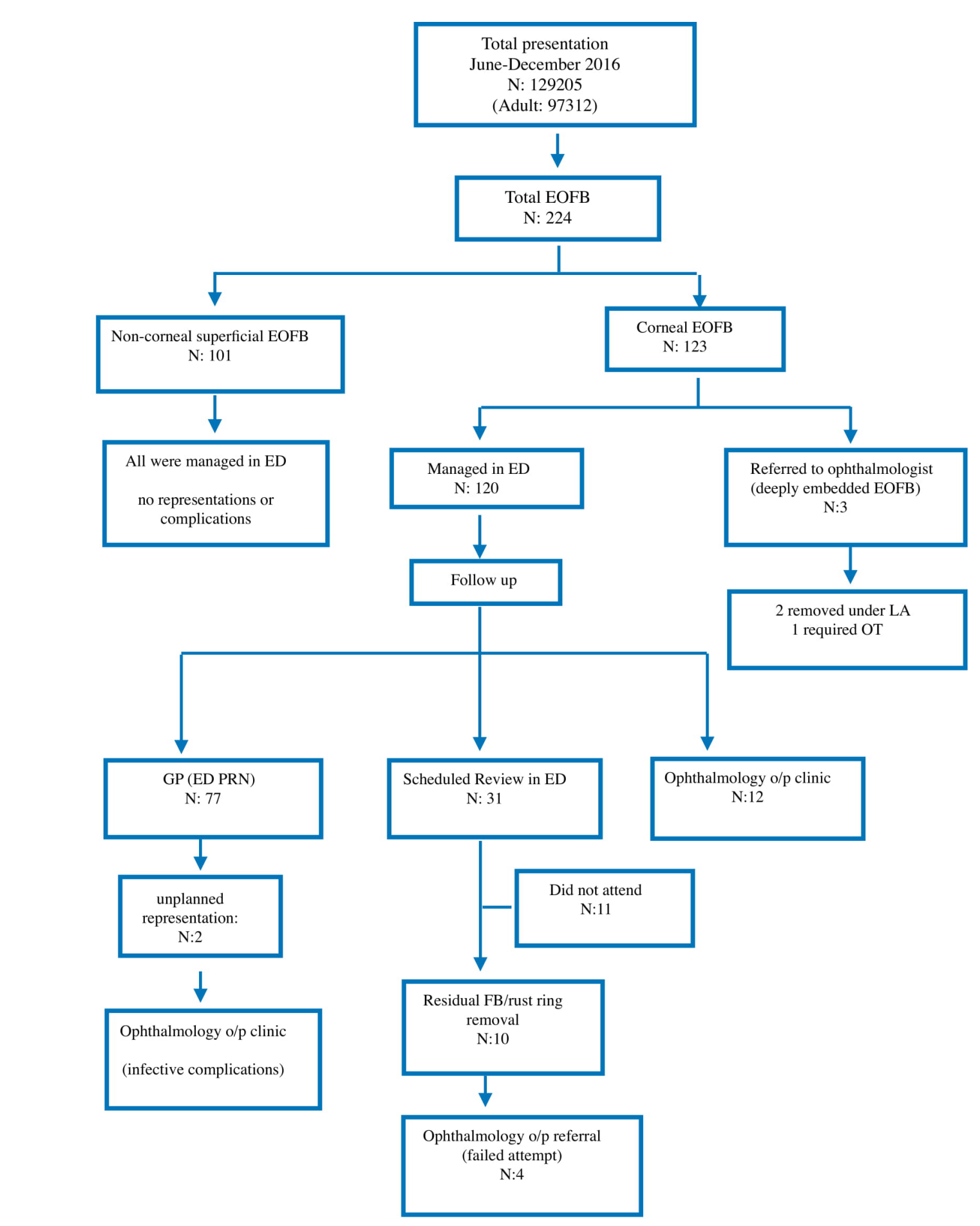

Figure 1: Study design flowchart.

\section{Material}

Out of a total of 123 patients, 26 (21.1\%) re-presented in the ED for ongoing symptoms or complications.

\footnotetext{
Of the 90 patients that presented with metallic EOFBs, 22
}

(25.3\%) re-presented, while none of the $4(0 \%)$ patients who had non-metallic EOFBs re-presented (Figure 2). 


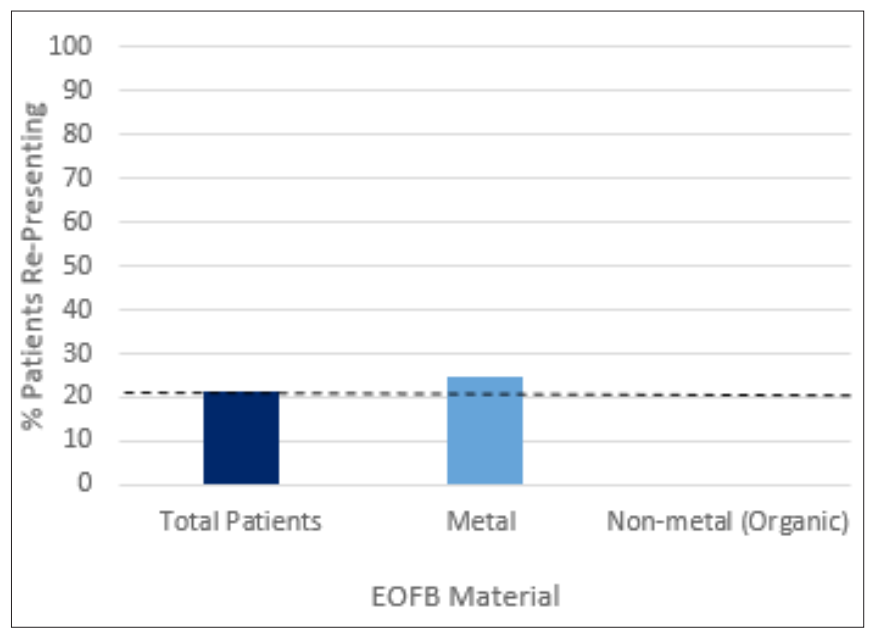

Figure 2: EOFB material and representation rate.

\section{Size (Figure 3)}

Patients with smaller EOFBs had a higher re-presentation rate, with 4 of 11 patients (36.3\%) with EOFBs of size $\leq 1-2 \mathrm{~mm}$ representing, of which 4 did so due to incomplete removal of EOFB at first attempt. This is in stark contrast to the 5 of $48(10.4 \%)$ patients who represented after having EOFBs of size $>1-2 \mathrm{~mm}$. (Figure 3).

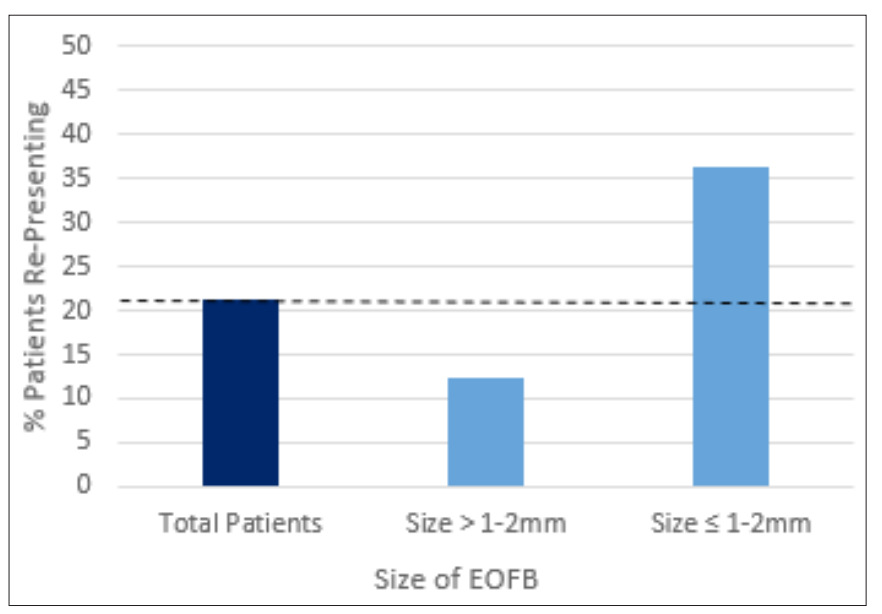

Figure 3: Size of EOFB and re-presentation rate.

\section{Visual acuity (Figure 3)}

Out of the 77 patients whose visual acuity at first presentation was documented, 22 had abnormal VA in the affected eye only and 16 in both the affected and unaffected eye. This translated to a higher re-presentation rate of 7 out of 22 (34.8\%) and 3 out of $16(18.8 \%)$ respectively. In contrast, only 3 of $39(7.7 \%)$ patients who had documented normal VA in the affected eyes re-presented (Figure 4). For the 13 patients who re-presented, the median VA in the affected eye was $6 / 9$, with the interquartile range of their VA being from $6 / 5$ to $6 / 24$ (Figure 5). There was no significant difference in the mean VA of patients who re-presented and did not re-present $(\mathrm{P}=0.0563)$.

\section{Age (Figure 4,5)}

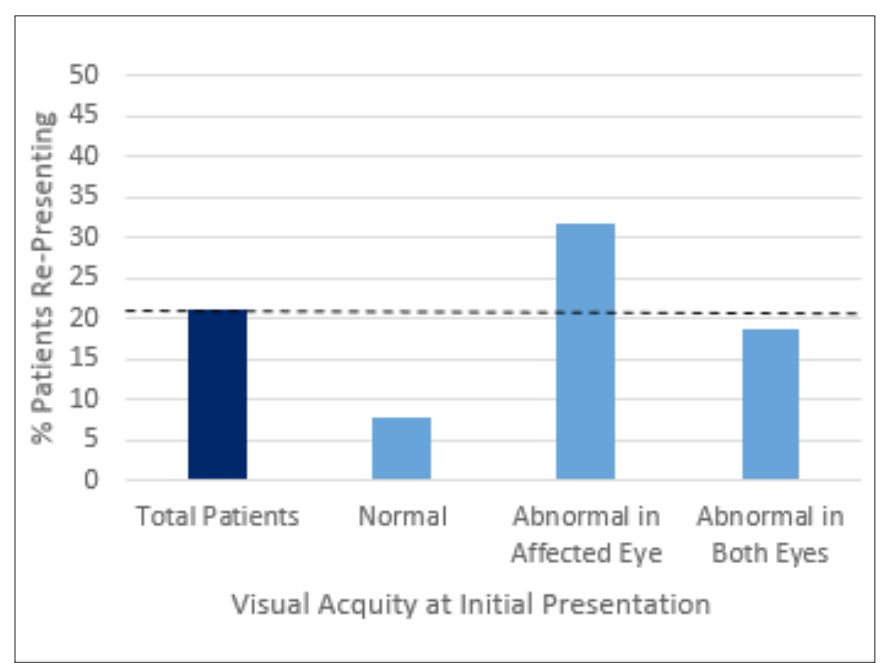

Figure 4: VA at initial presentation and re-presentation rate.

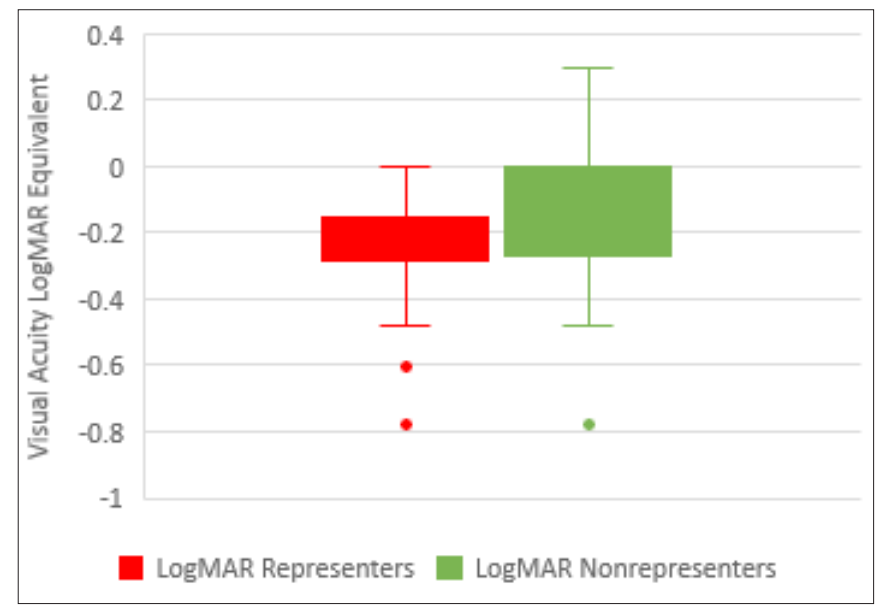

Figure 5: VA of patients with abnormal VA (in affected eye) on presentation.

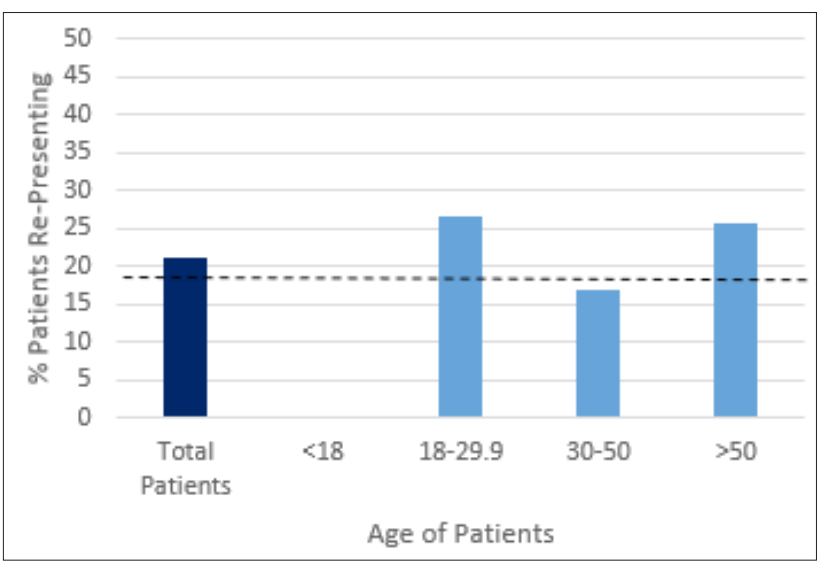

Figure 6: Age and re-presentation rate. 
8 of 30 patients (26.7\%) aged $18-30,10$ of 59 (16.9\%) patients aged $30-50$, and 8 of $31(25.8 \%)$ patients aged $>50$ re-presented to the ED either unscheduled or for a planned review (Figure 6). None of the 3 patients aged $<18(0 \%)$ re-presented to the ED. It was noted that 89 of 123 patients $(72.4 \%)$ of the study population were within the ages of 18-50 (Figure 6) (Table 1).

Table 1: Demography and univariate analysis of risk factors and re-presentation rate.

\begin{tabular}{|c|c|c|c|}
\hline Variable & Patients Re-presenting & Re-presentation rate (\%) & $\chi^{2} \mathbf{P}$-value \\
\hline Total Patients & 26 of 123 & 21.1 & - \\
\hline \multicolumn{4}{|c|}{ EOFB Material } \\
\hline Metal & 22 of 90 & 24.4 & 1 \\
\hline Non-metal & 0 of 4 & 0 & - \\
\hline \multicolumn{4}{|c|}{ Size of EOFB } \\
\hline$\leq 1-2 \mathrm{~mm}$ & 4 of 11 & 36.4 & 0.955 \\
\hline$>1-2 \mathrm{~mm}$ & 6 of 49 & 12.2 & 0.986 \\
\hline \multicolumn{4}{|c|}{ VA at First Presentation } \\
\hline Normal in affected eye & 3 of 39 & 7.7 & 0.777 \\
\hline Abnormal in affected eye & 7 of 22 & 31.8 & 0.97 \\
\hline Abnormal in both eyes & 3 of 16 & 18.8 & 0.777 \\
\hline \multicolumn{4}{|c|}{ Patient Age } \\
\hline$<18$ & 0 of 3 & 0 & - \\
\hline $18-30$ & 8 of 30 & 26.7 & 0.954 \\
\hline $30-50$ & 10 of 59 & 16.9 & 0.981 \\
\hline$>50$ & 8 of 31 & 25.8 & 0.954 \\
\hline
\end{tabular}

\section{Discussion}

With $21.1 \%$ of patients, either planned or unplanned, representing at the ED for ongoing symptoms or complications, it can be derived that a significant number of patients presenting with corneal EOFBs will need to be followed up and their condition cannot be resolved within the first intervention. Patients who were referred to the ophthalmology clinic for follow-ups mostly had residual EOFBs including rust rings and were unable to be removed by ED clinicians. Some patients also had complications such as infective keratitis post corneal abrasions, who required ophthalmologists' input. Most of the re-presentations were planned reviews, with the exception of 1 patient who developed infective keratitis following management in ED and another who was discharged with a small missed EOFB which was complicated by infective conjunctivitis.

Patients with metallic EOFBs appeared to have a higher representation rate following initial management, mostly due to secondary residual rust ring formation (a well-known complication of metallic FBs occurring hours after entrapment) as the contributing factor. The oxidisation of the offending metallic EOFB, usually iron, results in its diffusion into the corneal stroma's collagenous layers, where it can orchestrate an immune response which can not only delay healing but also lead to complications including chronic inflammation and corneal vascularisation [12]. As such, the complete removal of rust rings is usually performed, as was the case for a number of patients who re-presented. However, complete rust ring removal may not be achieved during a single consultation.
Widespread stromal application of a burr commonly used for rust ring removal can be detrimental and lead to scarring. When the rust ring is large and deep, it can be difficult to be completely removed without increasing the risk of excessive thinning and corneal damage. Peripheral rust may migrate superficially over time and slough off or be easier to remove, as such patients with large and deep rust rings may have to be scheduled for multiple follow ups to completely remove the rust. During the course of treatment, rust that does not stymie corneal healing can be left untouched $[13,14]$.

There is no census or recommendations as to how much residual rust can be left in place without any complications or cosmetic effects. This varies from patient to patient and is up to the discretion of individual ED physicians or ophthalmologists. Patients that presented with a smaller EOFB of size $\leq 1-2 \mathrm{~mm}$ had a significantly greater rate of re-presentation. Removal of corneal FBs can be a highly challenging procedure and can be made particularly more so by smaller FBs. Methods such as the use of a cotton bud lack the precision and fine motor control offered by the use of a syringe and needle, the classical removal method, which while more effective can be confronting for the patient and brings a higher risk of the clinician causing further damage or corneal perforation [15]. As such, complete removal of smaller EOFBs may be more difficult in the ED, resulting in more patients re-presenting or requiring follow-up by an ophthalmologist. It has been shown, initial visual acuity in intraocular foreign bodies, is a key prognostic and predicting factor, with a poor initial VA usually resulting in a poorer outcome [16]. This may also be applicable to EOFBs, given 
our observation that patients who had visual acuity deficits in the eye affected upon initial ED presentation, tended to re-present at a higher frequency than those who did not. Among the patients who had abnormal VA in the affected eye with EOFBs, there was no significant difference between the VA of these patients whether they re-presented or not. As such, we find that there is no range of VA deficit which can be used as an indicator of an increased risk of patient re-presentation.

It was observed that a significant number of patients fell within the ages of 18-50. This corresponds with the findings of other studies which found that the most common demographic for patients presenting with ocular foreign bodies in the ED are industrial workers of this age group, in particular industries where activities such as hammering metal or metal cutting is involved $[12,16-18]$. One study in Turkey found that a significant number of patients presenting to an eye clinic with metallic corneal foreign bodies worked in the metal industry, with $42 \%$ of the patients being aged 14-29 [19]. Among the patient age groups, it was also found that the age group with the most significant proportion of patients re-presenting was 18-30. People of the young adult age group, males in particular, have been found to have higher incidences of ocular trauma due to risk-taking behaviors and aforementioned industrial worker occupations, which may increase their chances of being afflicted with more severe eye injuries or getting metal EOFBs, which we have found to be a possible risk factor for representation $[10,20]$. However, given that age is an extremely broad category, we suggest that clinicians pay less heed to age and instead focus on other risk factors that patients may present with.

Given our findings, we propose that the following factors be taken into account for patients presenting with corneal EOFBs in the ED when making decisions on whether a patient should be referred for further review or management by the appropriate departments: Visual acuity at initial presentation, material and size of EOFB at initial presentation. Where the material of corneal EOFB is metal, size of EOFB is $\leq 1-2 \mathrm{~mm}$ or the patient presents with poor visual acuity at initial presentation, the patient would be considered to be at a higher risk of developing post-treatment complications or having unresolved conditions and more consideration should be given for follow-ups. We also suggest that in order to aid in better decision making and patient outcomes that ED clinicians document the EOFB size during their assessment.

\section{Limitations}

Given the nature of the study being retrospective, we were only able to review patient records as was documented and post discharge follow ups could not be carried out to collect further data. Taking into consideration that our study reviewed operational EDs where there exists no control over the type of patients presenting, patients of random variations would present with different conditions. This means that the sample size for each category within the risk factors studied may differ significantly. For example, among the patients only 3 were $<18$ years old while 61 were aged
30-50. This made it difficult to validate the data statistically given that the vast difference in sample size may result in bias towards a particular category or result. There were also other factors that could not be analyzed in the study due to a lack of data such as occupation, which could be a contributing factor to both the condition and/or complications. The small size of the study group could mean that the data may be partial to particular categories or impacted by unknown factors which skews the results. Adding on to that, patients in the study were all presenting only in hospitals in Melbourne, Victoria, which may not be representative of a larger population in different geographical locations, affecting the generalizability of the findings beyond our patient population. Further studies with a larger population of patients with measured EOFBs could be conducted to ascertain the validity of these findings.

\section{Conclusion}

This is the first study looking into the risk factors surrounding patient re-presentation following management of corneal EOFBs in the ED. Our study has identified that the following factors could potentially indicate an increased chance of patient re-presentation following discharge from the ED: EOFBs made of metal, size of EOFB $\leq 1-2 \mathrm{~mm}$, and abnormal VA at initial presentation. We suggest that ED clinicians adopt a more prudent approach in managing and referring out corneal EOFB patients by taking into account the aforementioned factors. Should the factors be identified by the ED clinician, an ophthalmologist's opinion should be obtained to guide patient management and review which could be done in the ophthalmology clinic or the ED. In the absence of the factors, the EOFB can likely be resolved in the ED and the patients should be instructed to return only if required as referrals for specialist management or scheduled reviews may be unnecessary for these patients. Due to the retrospective nature of the study and limited sample size, no definitive conclusions can be drawn on the risk factors of corneal EOFB re-presentations. Further prospective studies with a more diverse population and stricter documentation should be conducted to validate and emphasise these findings, so as to guide clinical practices.

\section{References}

1. Tielsch JM, Parver L, Shankar B (1989) Time trends in the incidence of hospitalized ocular trauma. Arch Ophthalmol 107(4): 519-523.

2. Bora C, Elisabeth JC, Rachel MC, Lisa P (2014) Epidemiology, clinical characteristics and complications in ocular foreign body injuries 55: 4713.

3. Pieramici DJ, Sternberg P, Aaberg TM, Bridges WZ, Capone A, et al. (1997) A system for classifying mechanical injuries of the eye (Globe). Am J Ophthalmol 123(6): 820-831.

4. Cascairo MA, Mazow ML, Prager TC (1994) Pediatric ocular trauma: A retrospective survey. J Pediatr Ophthalmol Strabismus 31(5): 312-317.

5. Sternberg P, Juan E de, Michels RG, Auer C (1984) Multivariate analysis of prognostic factors in penetrating ocular injuries. Am J Ophthalmol 98(4): 467-472.

6. Andrew AD (2017) Conjunctival foreign body removal. 
7. International Centre for Eye Health (2005) Ophthalmic practice. Community Eye Heal J 18(55): 109-110

8. Melton R, Thomas R (2004) Corneal foreign body. Clin Refract Optom 15(11-12): 318-323

9. Raymond S, Favilla I, Nguyen A, Jenkins M, Mason G, et al. (2009) Eye injuries in rural Victoria, Australia. Clin Experiment Ophthalmol 37(7): 698-702.

10. Woo JH, Sundar G, Ophth A (2006) Eye injuries in Singapore-don't risk it. Do More. A Prospective Study. Ann Acad Med Singap 35(10): 706-718.

11. Jack TH (1997) Proper method for calculating average visual acuity. J Refract Surg 13(4): 388-391.

12. Zuckerman BD, Lieberman TW (1960) Corneal rust ring: Etiology and histology. AMA Arch Ophthalmol 63(2): 254-265.

13. Guier CP, Stokkermans TJ (2020) Cornea foreign body removal. Stat Pearls, USA.

14. Aaron BO (2017) No insult to injury: Managing foreign body removal Rev Optom.
15. Beyer H, Cherkas D (2012) Corneal foreign body removal using a bent needle tip. Am J Emerg Med 30: 489-490.

16. Greven CM, Engelbrecht NE, Slusher MM, Nagy SS (2000) Intraocular foreign bodies: Management, prognostic factors, and visual outcomes. Ophthalmology 107(3): 608-612.

17. Meng ID, Kurose M (2013) The role of corneal afferent neurons in regulating tears under normal and dry eye conditions. Experimental Eye Research 117: 79-87.

18. Aziz MA, Rahman MA (2004) Corneal foreign body--an occupational hazard. Mymensingh Med J 13(2): 174-176.

19. Ozkurt ZG, Yuksel H, Saka G, Guclu H, Evsen S, et al. (2014) Metallic corneal foreign bodies: An occupational health hazard. Arq Bras Oftalmol $77(2): 81-83$.

20.Zagelbaum BM, Tostanoski JR, Kerner DJ, Hersh PS (1993) Urban eye trauma: A one-year prospective study. Ophthalmology 100(6): 851-856.

For possible submissions Click below: 